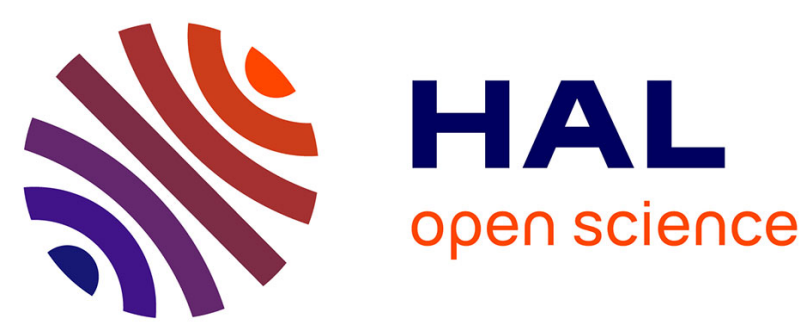

\title{
Les habitats Hallstattiens de Chinon (Indre-et-Loire) Gérard Cordier
}

\section{To cite this version:}

Gérard Cordier. Les habitats Hallstattiens de Chinon (Indre-et-Loire). Gallia - Fouilles et monuments archéologiques en France métropolitaine, 1985, 43 (1), pp.1-23. 10.3406/galia.1985.2819 . hal-01935188

\section{HAL Id: hal-01935188 \\ https://hal.science/hal-01935188}

Submitted on 4 Feb 2020

HAL is a multi-disciplinary open access archive for the deposit and dissemination of scientific research documents, whether they are published or not. The documents may come from teaching and research institutions in France or abroad, or from public or private research centers.
L'archive ouverte pluridisciplinaire HAL, est destinée au dépôt et à la diffusion de documents scientifiques de niveau recherche, publiés ou non, émanant des établissements d'enseignement et de recherche français ou étrangers, des laboratoires publics ou privés.

\section{(이) $\$$}

Distributed under a Creative Commons Attribution - NonCommercial - NoDerivatives| 4.0 


\title{
LES HABITATS HALLSTATTIENS DE CHINON (Indre-et-Loire)
}

\author{
par Gérard CORDIER
}

On doit à la sagacité d'un entrepreneur de maçonnerie chinonais, M. Jean Zocchetti, la révélation d'une série d'habitats hallstattiens dans la vallée de la Vienne, un peu en amont de Chinon (fig. 1). Quelques-uns de ces sites, qui livrèrent parfois aussi des vestiges gallo-romains, ont été succinctement signalés depuis une trentaine d'années, tant dans les Informations de Gallia (Cordonnier-Détrie 1955), que dans les bulletins des Amis du Vieux Chinon (Sacchi et Zocchetti 1965, 1967 ; Amis du Vieux Chinon, 1967, 1968, 1981). Mais il restait à produire l'étude d'ensemble que méritait un matériel céramique abondant el d'un intérêt indéniable, bien que recueilli fortuitement à l'occasion de travaux de terrassement et le plus souvent très fragmentaire. Tel est le but de la présente publication, qui n'aurait pu être réalisée sans la libéralité de M. J. Zocchetti.

Les sites seront décrits de l'ouest à l'est (les numéros renvoient au plan de la fig. 1). La présentation topographique sera simplifiée par la similitude des lieux, puisque tous se localisent dans la bande de terrain relativement étroite comprise entre la plaine inondable de la Vienne et les coteaux turoniens exposés au midi qui la bordent sur sa rive droite. Les sites se succèdent sur une longueur de $700 \mathrm{~m}$. Ils sont distants de la rivière de 800 à $1200 \mathrm{~m}$; leurs altitudes N.G.F. se situent entre 34 et $40 \mathrm{~m}$, l'étiage de la Vienne étant d'environ $30 \mathrm{~m}$ et le niveau du lit majeur variant de 31 à $33 \mathrm{~m}$.

\section{Les Bas de Sainte-Radégonde. Site 1.}

C'est en 1967, lors de la construction de la maison Zocchelti Angèle (cadastre A'l' 44), à $80 \mathrm{~m}$ au nord de la rue Paul-Huet, près de la voie ferrée, que fut découvert le matériel le plus important. La surface explorée comprend le sous-sol de la maison prolongé jusqu'à une douzaine de mètres vers le sud par quatre tranchées d'adduction d'eau. Le niveau archéologique s'étend sur toute cette surface à une profondeur de 0,70 à $0,80 \mathrm{~m}$ et avec une épaisseur de 0,15 à $0,20 \mathrm{~m}$; il est constitué d'un sable limoneux brun, riche en débris de poterie, présentant des passées de terre et de pierres brûlées, sans toutefois qu'il ait 

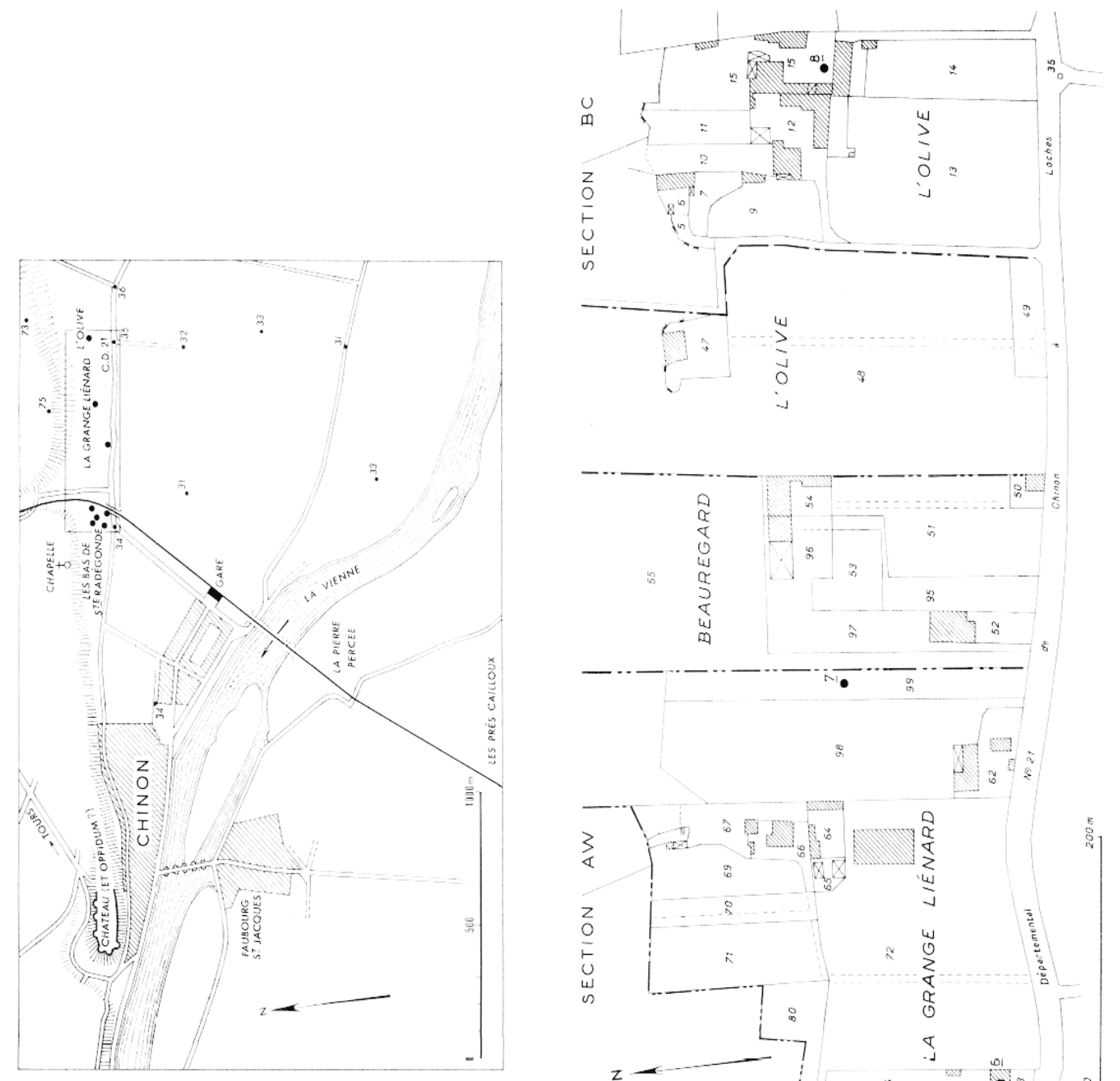

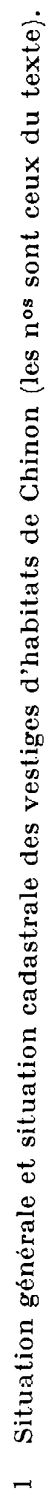
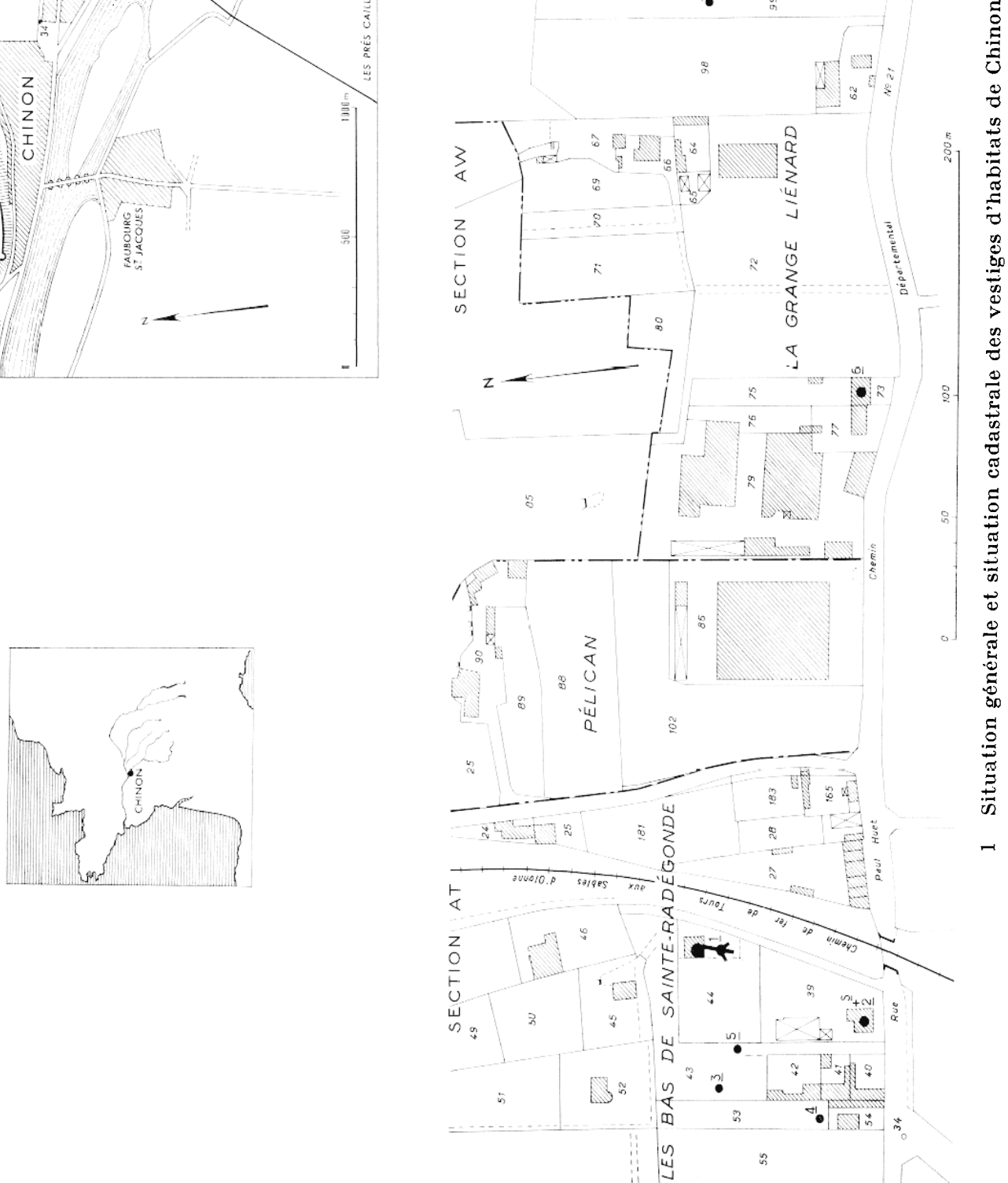
été rencontré de véritable foyer. Le matériel, qui semble pouvoir être traité comme un ensemble, se répartit comme suit :

1. Céramique relativement grossière, non lissée, à surface plus ou moins rugueuse, pâte contenant un dégraissant de grains de quartz et de particules calcaires et présentant parfois des vacuoles dues à des débris organiques disparus, surface de lons marron clair à noirâlre ; le montage au colombin est souvent attesté par l'aspect de surface comme par l'orientation des cassures. La cuisson, excellente et généralement homogène, a donné des vases relativement solides. Le matériel est néanmoins très fragmentaire ; la plupart des cassures sont anciennes. Il comprend quelque 520 tessons mesurant de 2 à une vingtaine de centimètres dans leur plus grande dimension, dont certains ont permis la reconstitution de portions importantes de vases de grand gabarit¹.

Jarre à panse régulièrement galbée, ovoïde, remontée presque entièrement, à l'exception du col (fig. 2). Surface marron clair, présentant des traces d'ébauchoir en tous sens à l'extérieur et des desquamations à l'intérieur. Quatre trous de réparation bien réguliers de 4 à 6 mm de diamètre. D.G. : 420 ? ; D.P. : 505 ; D.F. : 210 ; H. : (567) ; e. : 12 en moyenne sur les flancs, 20 au voisinage du fond, 16 à 19 au fond.

Portion d'environ un quart du pourtour d'une jarre à panse irrégulièrement galbée (fig. $3, n^{\circ} 3$ ), col éversé, ouverture évasée à bord largement festonné, décor consistant en un cordon à impressions digitales courant à environ $50 \mathrm{~mm}$ du bord. Surface brun noirâtre. D.O. : 484 ; D.C. : 464 ; D.P. : 516 ; H. : (450); e. : 9 à 13 , 15 au cordon.

Portion inférieure d'un vase de forme probablement voisine des précédentes, à profil irrégulier, fond faiblement concave et légèrement débordant (fig. $3, \mathrm{n}^{\circ} 2$ ). Surface presque lisse, de ton beige avec passées noirâtres. D.F. : 190 ; c. : 8 à 10 aux flancs, 12 à 20 au fond.

Ces reconstitutions effectuées, il reste un lot de 450 tessons dont les épaisseurs se répartissent ainsi : 5 à $10: 61,2 \% ; 10$ à $15: 37,9 \%$ \% ; 15 à $20: 0,9 \%$. 68 d'entre eux fournissent des indications de formes ou de décors.

Les Formes présentent des :

cols cylindriques : 4 cas (fig. $4, n^{\text {os }} 2,6,7$ et fig. $5, \mathrm{n}^{\circ} 12$ ) ; 2 estimations dimensionnelles possibles : D.O. : 240 et 380 (fig. 4 , nos 2 et 7 );

cols tronconiques ouverts : 9 cas (fig. $4, n^{0} 10$; fig. 5 , nos 1,8 et fig. 6, n $^{\text {os }} 5,7$ à 10 ); une estimation possible : D.0. : 500 (fig. $5, \mathrm{n}^{0} 1$ );

cols évasés : 10 cas (fig. $4, n^{0} 1$ et fig. $6, n^{\text {os }} 12$ à 20$) ; 2$ estimations possibles : D.O. : 240 et D.G. : 210 (pour le même vase, fig. $6, \mathrm{n}^{0} 20$ ) ;

bords de bols ou de terrines à profil convexe : 4 cas (fig. 6, nos $^{\circ}$ à 4 );

fonds plats avec départ de llancs plus ou moins obliques : 9 cas (fig. $6, n^{\text {os }} 21$ à 29 ), dont 2 franchement débordants (fig. 6 , nos 22 et 24 ) ; 2 estimations possibles : D.F. : 105 et 110 (fig. 6, nos 27 et 29 ).

1 Abréviations employées : D.O. : diamètre ouverture; D.C. : diamètre col ; D.P. : diamètre panse; 1).J. : diamètre fond ; H. : hauteur ; e. : épaisseur. Beaucoup de ces dimensions ont élé évaluées par extrapolation et doivent être considérées comme approximatives. Sauf indication contraire, toutes les dimensions sont données en millimètres. Pour la terminologie céramique, le présent travail a beaucoup emprunté à B. Chertier (1976). 


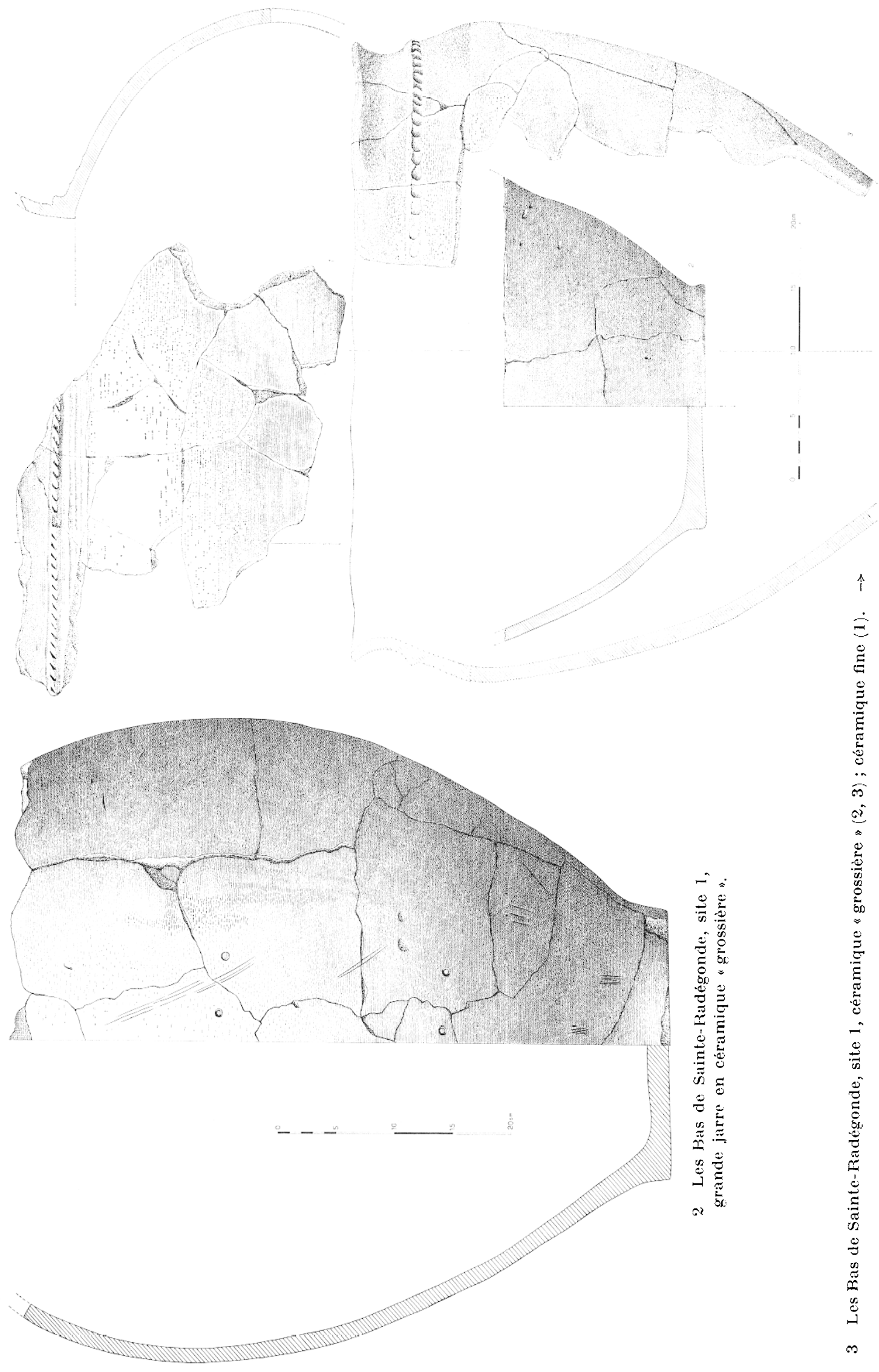


Bon nombre de fragments de cols et de fonds peuvent provenir de grandes jarres comme celles qui ont pu être en partic reconstituées. Un fragment se rapporte à un vase de type unique dans notre matéricl : un gobelet ou grosse tasse à anse (fig. $\left.5, n^{\circ} 4\right)$, à surface presque lisse, de teinte marron clair avec passées sombres et parsemée de particules de mica ; la pièce présente un trou de réparation maladroitement foré.

Décors. Ils sont soit localisés sur l'épaule des vases ou à la rupture du col (lignes d'impressions digito-unguéales ovalaires (fig. $4, \mathrm{n}^{\circ} 2$ et fig. $5, \mathrm{n}^{\circ \mathrm{s}} 10,12$ ) ; cordons plus ou moins saillants avec impressions de même type (fig. $4, n^{\text {os }} 3,9,11,13,14,15,18$ et fig. 5 , $\mathrm{n}^{0} 1$ ); cordons à impressions à la spatule (fig. $\left.4, \mathrm{n}^{\mathrm{os}} 16,17,20,21\right)$; cordon, à décor d'aspect torsadé (fig. 4, nos $6,7,12)$ ) ; soit localisés sous des rebords de jarres, de bols ou de terrines (impressions digitales (fig. 4, no 10 ); impressions exécutées à l'aide d'un instrument à extrémité triédrique (fig. $\left.\overline{5}, \mathrm{n}^{\circ} 3\right)$ ).

Les décors sur les lèvres présentent des incisions obliques (unguéales?) sur lèvre arrondic (fig. $4, \mathrm{n}^{\circ} 19$ ); impressions digitales sur lèvre arrondie (fig. $4, \mathrm{n}^{\circ} 1$ et fig. $5, \mathrm{n}^{\circ} 9$ ) ; impressions digitales sur lèvre épaissie, formant bourrelet extérieur ou intérieur (fig. 5 , $\mathrm{n}^{\text {os }} 5,8,11$ ); cannelures obliques sur lèvre aplatie (fig. $5, \mathrm{n}^{\circ} 1$ ).

Décors internes. Un tesson provenant d'une grande jarre présente des impressions digitales larges et peu profondes alignées au voisinage de la lèvre; il semble difficile de décider s'il s'agit d'un décor intentionnel ou de traces de façonnage (fig. $5, n^{\circ} 1$ ).

2. Céramique fine, lustrée, de tons noirâtre ou grisâtre.

La pièce la plus intéressante de ce lot est une élégante coupelle surbaissée, carénée, à ouverture très évasée, fond arrondi et ombiliqué, décor de 2 cannelures jointives à la carène et traces d'un décor interne peint ou appliqué consistant en chevrons formant 8 motifs de triangles emboîtés, orientés sommets vers le centre (fig. 7, no 3). D.0. : 110 ; D.C. : 85 ; I.P. : 87 ; H. : 45 ; ombilic de 13 à 14 de diam. et de 1 à 2 de prof. ; c : 4 à 5) au col, 2,5 à 3 à la panse, 5 au fond. 6 tessons à décor de 2 cannelures jointives proviennent d'au moins 3 coupelles de ce type (fig. $7, \mathrm{n}^{\text {os }} 1,2,9,10,12$ ) ; l'un d'eux présente les traces d'un décor interne analogue au précédent (fig. $7, \mathrm{n}^{0} 2$ ).

Outre ces pièces, le lot comprend environ 80 tessons dont les épaisseurs se répartissent ainsi : 5 à $10: 88 \% ; 10$ à $12: 12 \%$. Une trentaine d'entre eux permettent les observations suivantes :

Les Formes présentent des:

cols tronconiques ouverts : 4 cas (fig. $8, \mathrm{n}^{\text {os }} 1,16,17$ );

cols évasés : 6 cas (fig. 8 , nos $2,3,13,14,15$ ); dans 2 cas, le bord est déjeté à l'extéricur (fig. $8, \mathrm{n}^{\text {os }} 2,3$ ), ces derniers proviennent probablement d'urnes de D.0. : 225 et 280 ;

bords de bols ou de terrines : 7 cas (fig. 7, n$^{\circ} 15$ et fig. 8 , nos 4 à 9 ); 2 d'entre cux accusent un I).0. de 220 (fig. 8 , nos 6,7 );

bords de jattes : 2 cas (fig. 7, n $\left.^{\text {os }} 4,5\right)$;

fond plat: 1 fragment avec départ de flanc très oblique (fig. 8 , no 18) ; D.F. : 116.

Les Décors. Des stries avant cuisson : 2 cas, en séries de 2 et 3 (fig. 7, nos 8,17 ); des stries après cuisson : 1 cas, en séries de 5 , internes, rayonnantes (fig. $7, n^{\circ} 4$ ); des cannelures 

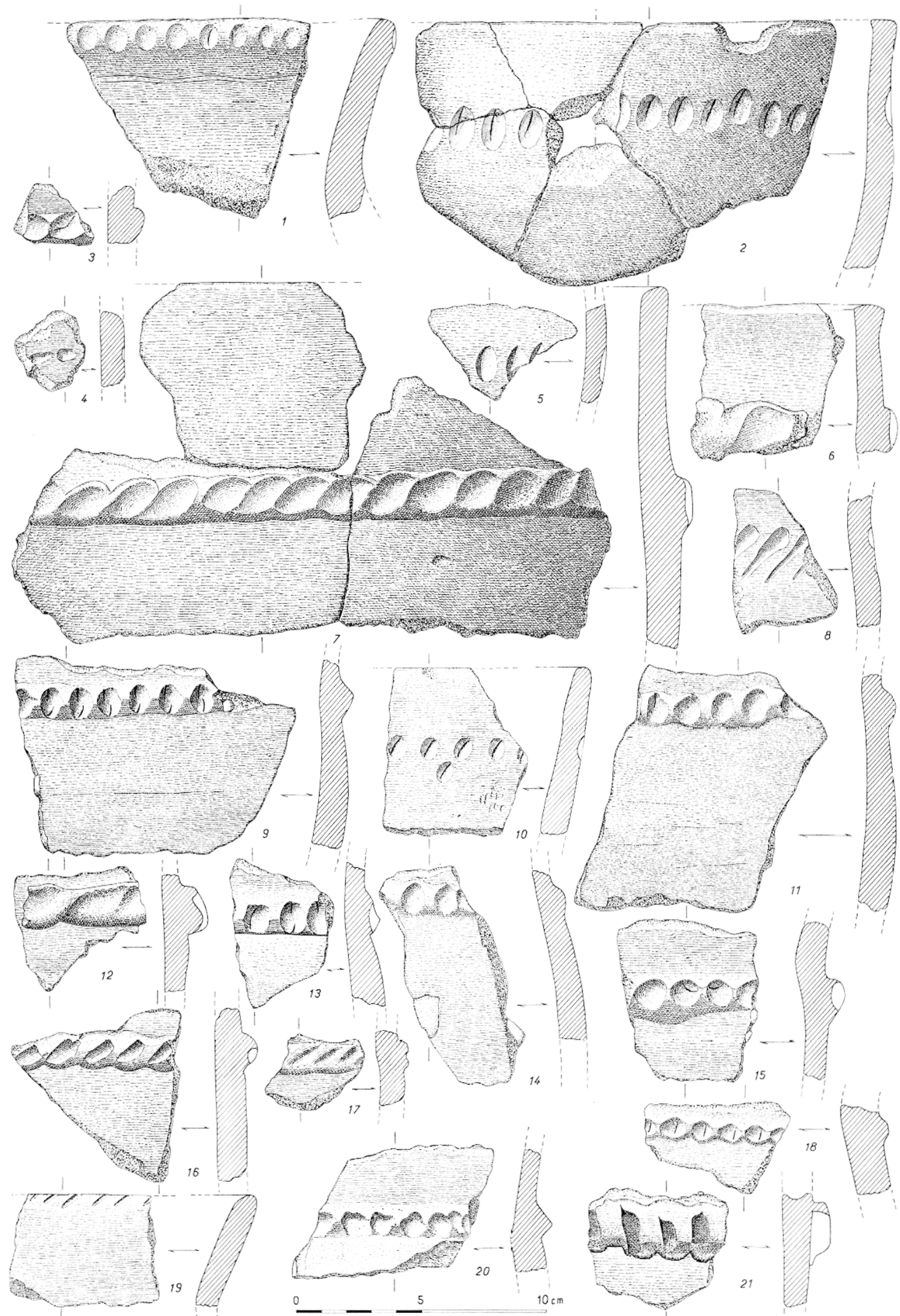

4 Les Bas de Sainte-Radégonde, site l, céramiqque "grossière ", tessons à impressions diguitales col aul.res. 


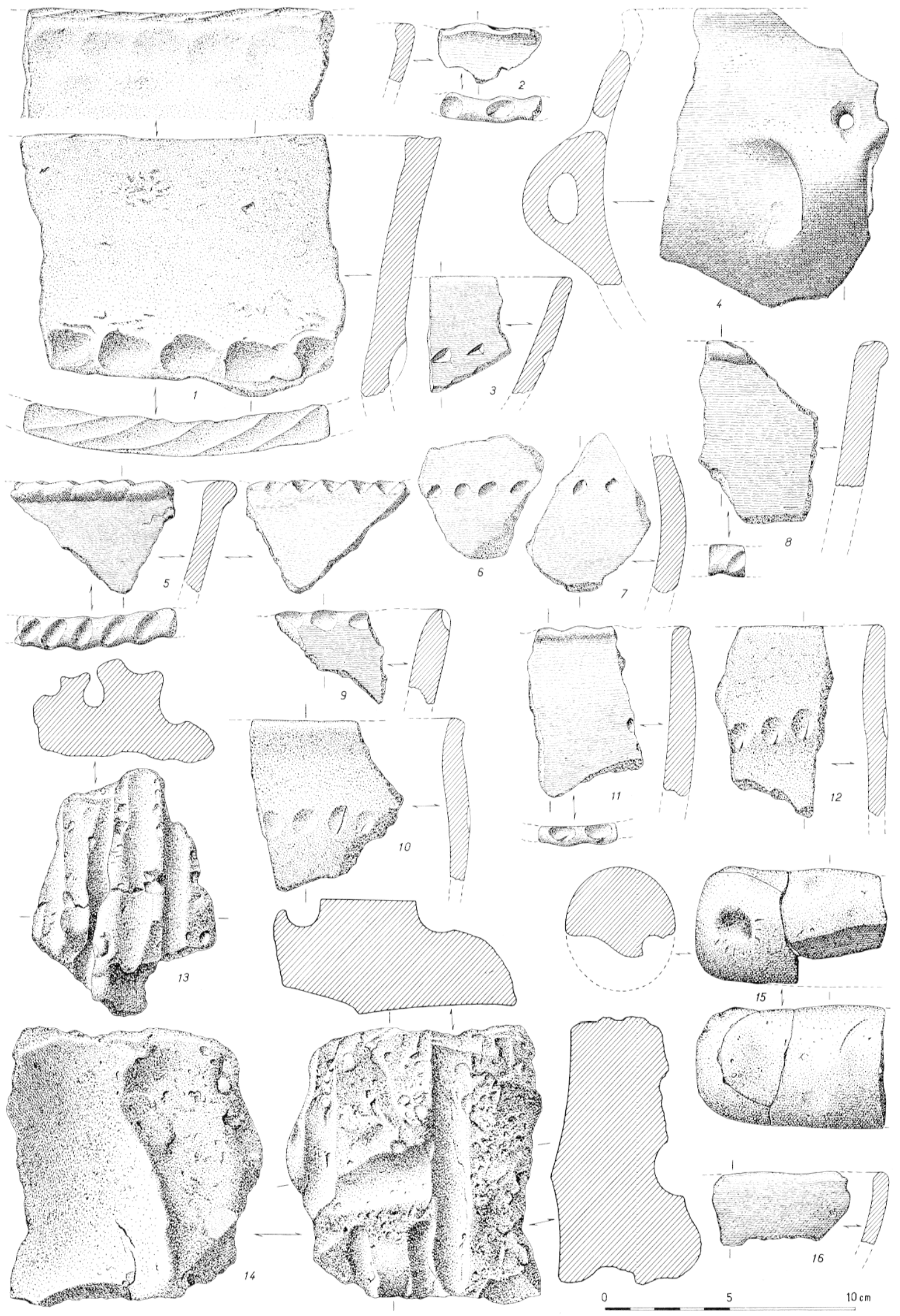

5 Les Bas de Sainte-Radégonde, site 1, céramique "grossière ", tessons à impressions diverses, fragment de gobelet à anse (4), débris de pisé $(13,14)$, fragment d'objet indéterminé (15). 


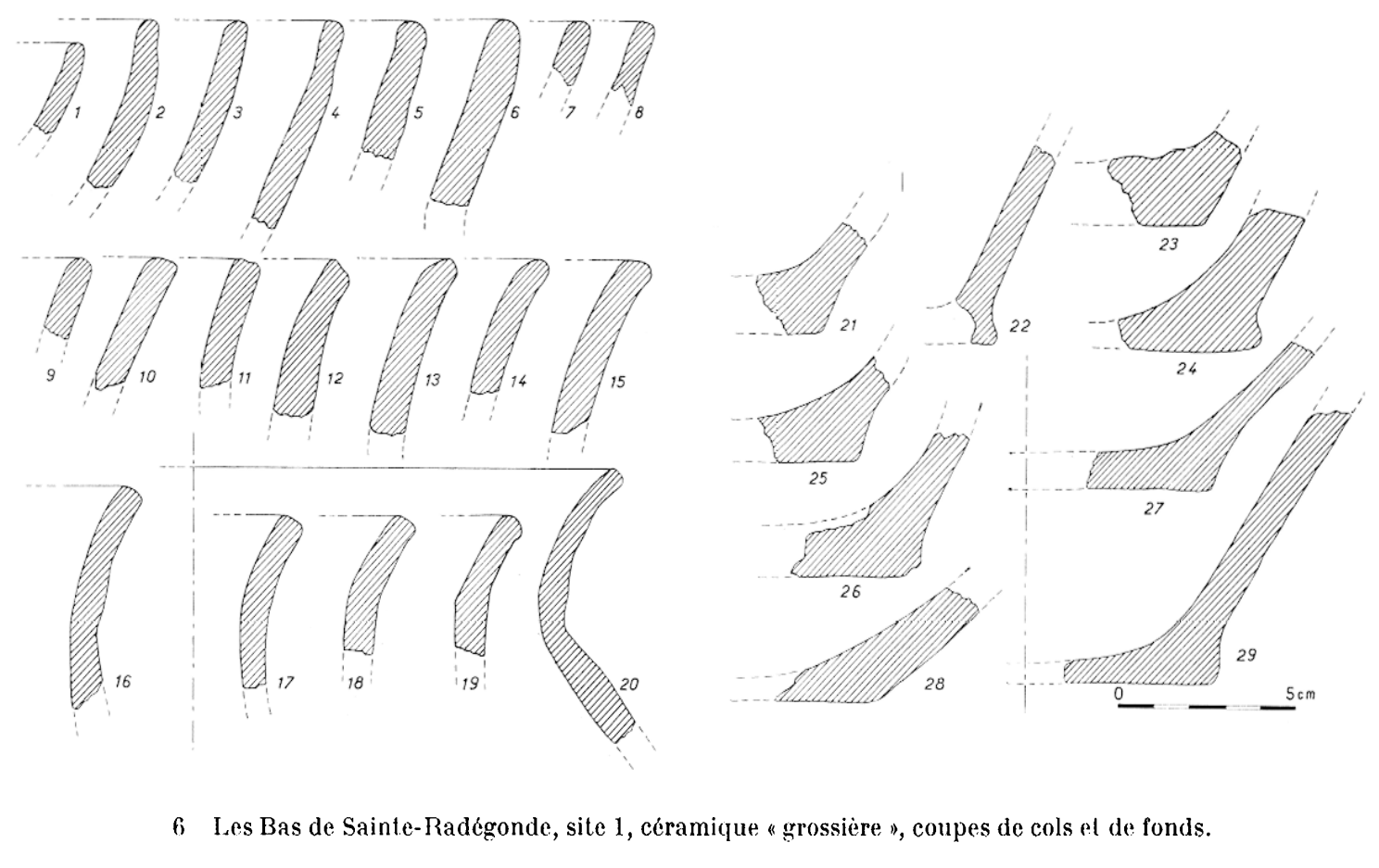

étroites ( 2 à 3 de largeur) : 1 série de 2 et 1 de 4 (fig. $7, n^{\text {os }} 7,16$ ); des cannelures larges ( 4 à 8 de largeur), jointives : 3 cas, en séries de 2 et 3 (fig. $7, n^{\text {os }} 6,11,13$ ); les bords moulurés intérieurement : 3 cas (fig. 7, nos $^{\circ}, 5$ et fig. $8, n^{\circ} 2$ ).

3. Céramique lisse, lustrée, à pâte assez fine, de bonne cuisson, solide, surface de tons beige ou marron clair passant parfois au brun rougeâlre.

Ce lot, peu important mais assez homogène, comprend :

unc portion de grosse urne bulbeuse à col éversé, reconstituée avec une douzaine de tessons à surface de ton marron avec passées noirâtres, traces d'ébauchoir horizontales (fig. 3, no 1). Décor composé d'un cordon à impressions obliques situé à la rupture du col, surmontant une série de 4 cannelures jointives peu profondes de 7 à 10 de largeur ; une autre cannelure, de 4 à 5 de largeur, court au-dessus du cordon. D.0. : >550; D.C. : 525 ; D.P. : 840 ; e. : 9 à 12,17 au cordon ;

un tesson décoré d'un cordon à impressions ovalaires obliques souligné de 2 stries (fig. $7, \mathrm{n}^{\circ} 14$ ); e. : 6,5 à 8,5 ;

un fragment de terrine à décor de 2 cannelures (fig. 7 , no 18) ; D. $0 .: 168$; D.P. : 174 ; e. : 4 à 6 ;

un fragment de terrine à bord légèrement rentrant, décoré de 3 cannclures (fig. 7, no 19); D.O. : 198 ; D.P. : 210 ; e. : 5 à 7 ;

un fragment de col tronconique franchement déjeté vers l'extérieur (fig. $8, \mathrm{n}^{\circ} 1$ ); D. $0 .: 240$; e. : 6 à 7 ;

Quelques débris inutilisables. 


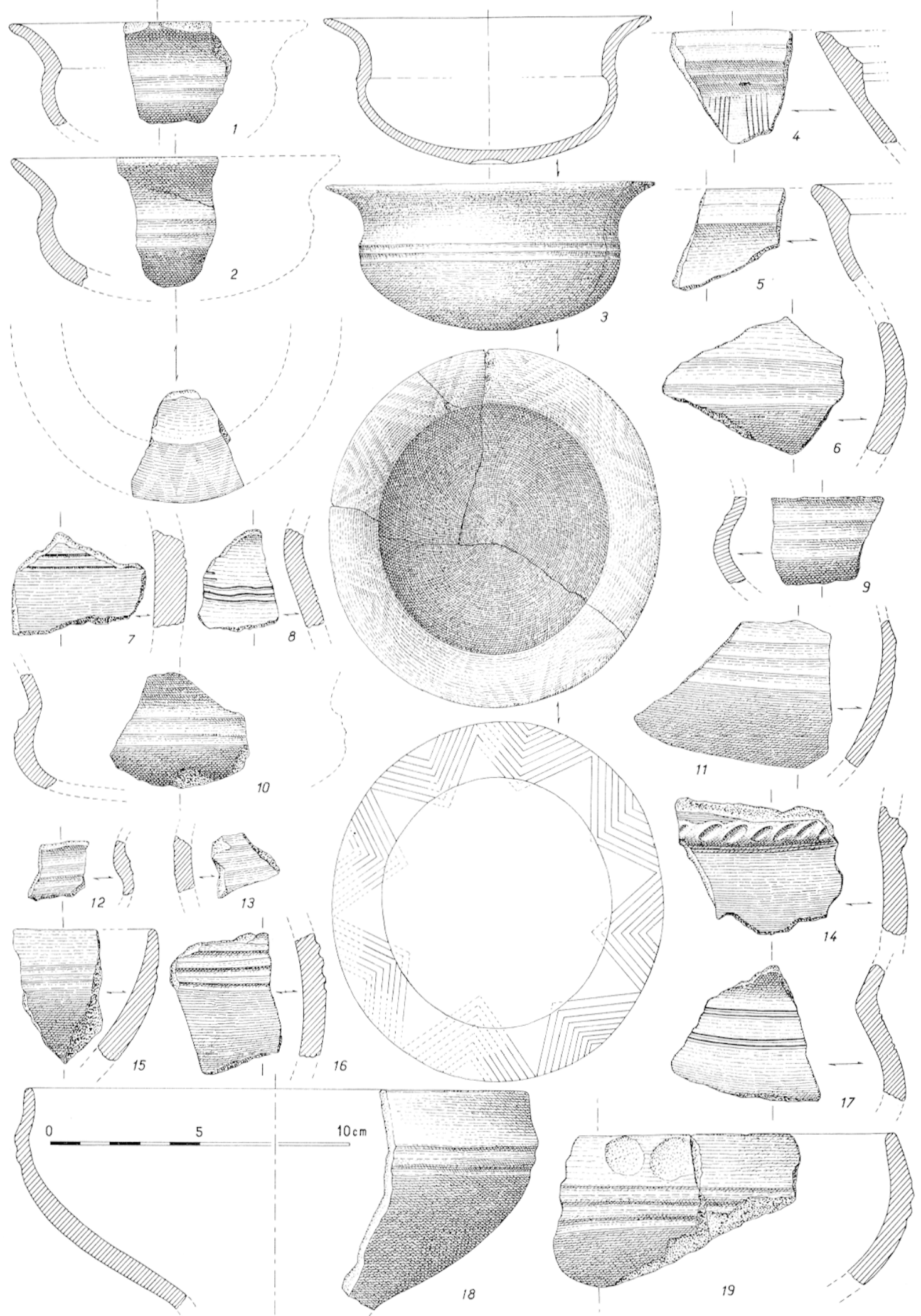

7 I.es Bas de Sainte-Radegonde, sile 1, círamique fine, coupelles (1 à 3), terrines (18, 19), tessons à décors de cannelures et d'incisions. 


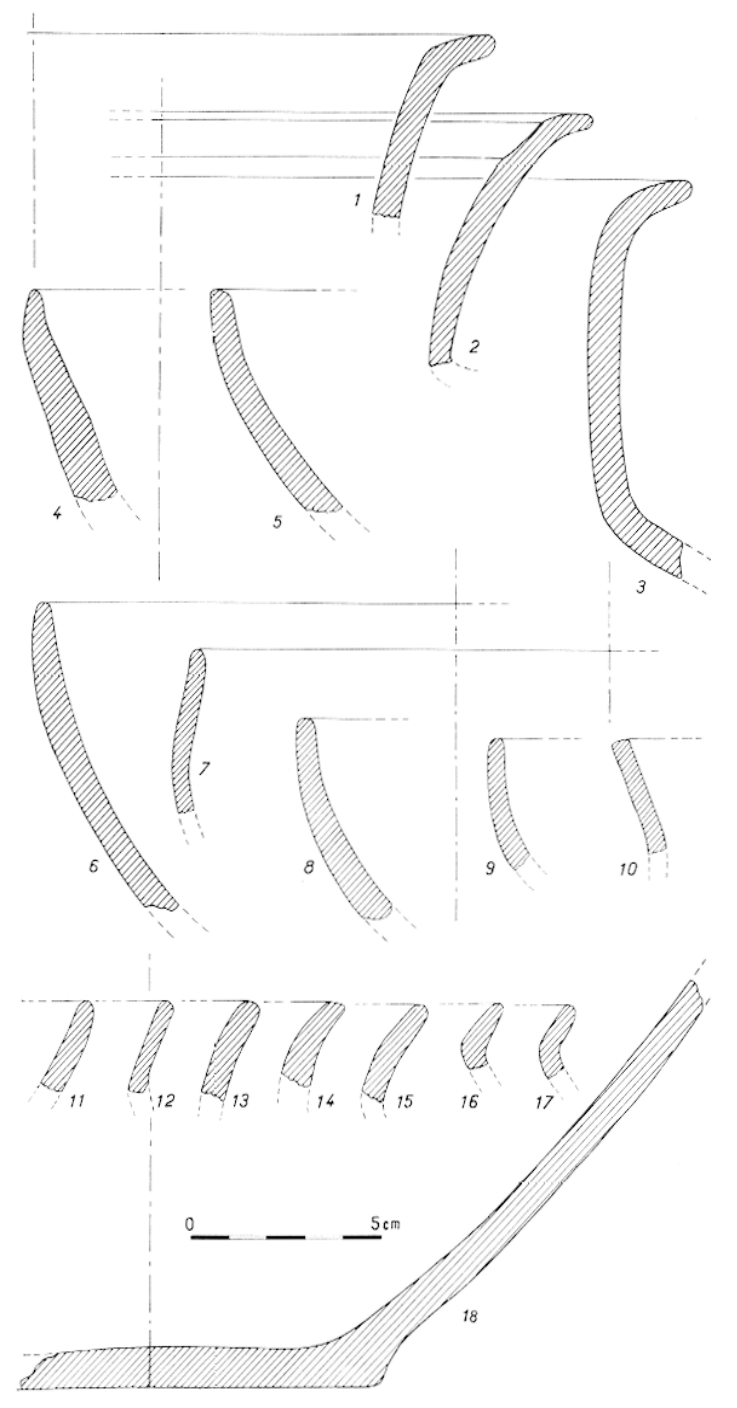

8 Les Bas de Sainte-Radégonde, site 1, céramique fine, coupes de cols et de fond d'urnes, de jattes et de terrine.
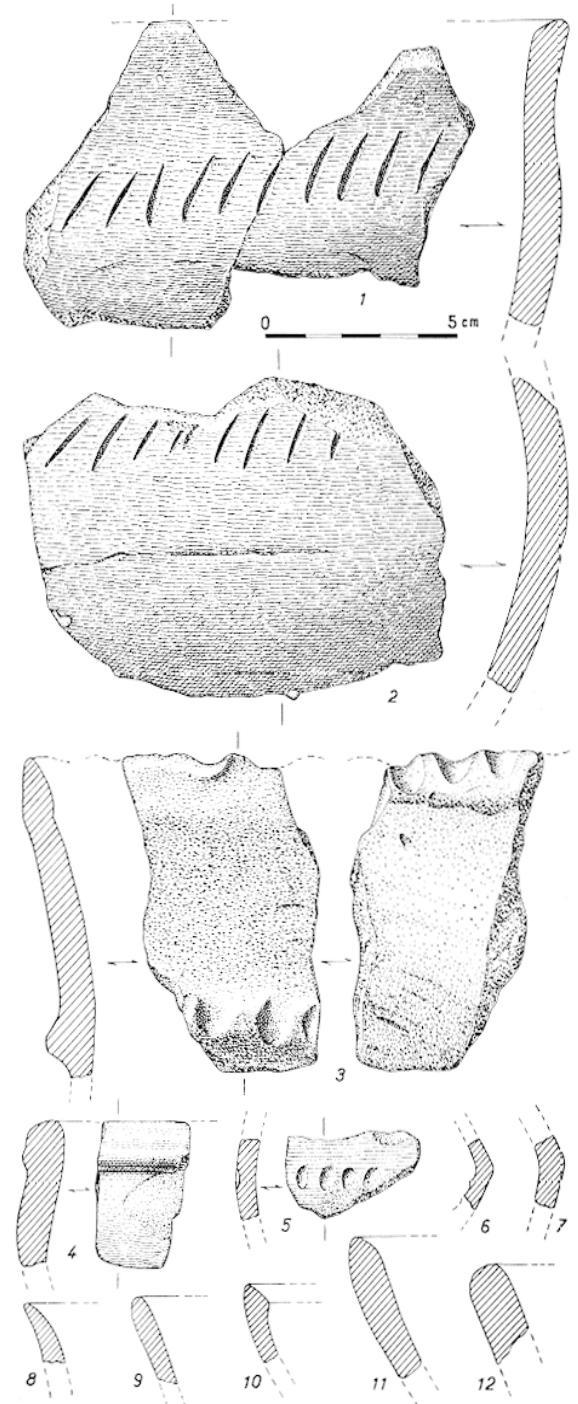

9 Les Bas de Sainle-Radégonde, site 2, céramique "grossière " $(1,2)$. Sile 3 , céramique "grossière " $(3,4)$; céramique fine $(5$ a 12$)$.

4. Débris de pisé grisâtres, d'aspect cendreux, avec empreintes de clayonnages parallèles et perpendiculaires : une quinzaine d'échantillons (fig. 5, nos 13,14 ).

5. Indéterminé : un objet cylindrique incomplet de 75 de longueur et 45 de diamètre, à extrémité arrondic, présentant une cavité sur le côté (fig. $5, n^{\circ} 15$ ); peut-être s'agit-il d'une poignée de vase, de lampe ou de creuset?

6. Mâchefer : quclques rares fragments.

7. Faune : quelques débris osseux et dents (sanglicr, pore?). 
Laes Bas de Sainte-RadF́gonde. Site 2

En 1949, lors du creusement des fondations de la maison Zocchelli .Jean (cadastre AT 39), 36 rue Paul-Huel, soit à $70 \mathrm{~m}$ au sud - sud-ouest du point précédent, fut rencontré, sous une couche d'environ $0,40 \mathrm{~m}$ d'épaisseur riche en tessons gallo-romains, un niveau de sable limoneux brun de 0,15 à $0,20 \mathrm{~m}$ d'épaisseur, qui livra :

2 tessons non raccordables provenant d'un grand vase à panse galbéc et col légèrement éversé, de pâte beige non lissée mais peu rugueuse, décoré d'incisions obliques (fig. $9, \mathrm{n}^{\text {os }} 1$, 2) ; D.P. : 500 ; c. : 8 à 11 ;

quelques éclats de silex informes, bruts, et un nucléus médiocre, irrégulier (Sacchi et Zocchetti 1965).

$\mathrm{A} 2 \mathrm{~m}$ à l'est de la maison (point $\mathrm{S}$ du plan) fut découverte une sépulture sans structure de protection ni mobilier, comprenant un squelette complet, allongé sur le dos, orienté est-ouest.

\section{Les bas ide Sainte-Radégonde. Site 3}

A $55 \mathrm{~m}$ à l'ouest du premier point (jardin Moreau, cadastre AT 43), en octobre 1967, un petit terrassement de $1,50 \times 1,50 \mathrm{~m}$ a mis au jour à la même profondeur le même niveau archéologique et livré le matériel suivant:

1. Céramique "grossière»: une cinquantaine de tessons de 8 à 15 d'épaisseur, parmi lesquels 4 fragments de cols dont un décoré d'impressions digitales sur la lèvre et sur un cordon (fig. $9, n^{0} 3$ ), ainsi qu'un autre décoré près du bord d'un bourrelet horizontal surmontant 2 stries (fig. 9 , no 4 ).

2. Céramique fine, lustrée, noirâtre : une douzaine de tessons de 5 à 7 d'épaisseur, dont un à impressions ovalaires (fig. $9, \mathrm{n}^{\circ} 5$ ), un à cannelure (fig. $9, \mathrm{n}^{\circ} 6$ ) et 5 débris de rebords (fig. 9, n $^{\text {os }} 8$ à 12 ).

3. Silex : un mauvais nucléus en fin de débitage et petit éclat brut.

\section{Les Bas de Sainte-Radégonde. Site 4}

A $40 \mathrm{~m}$ au nord-ouest de la maison Zocchetti Jean (maison Dion, cadastre AT 53), en septembre 1967, le creusement d'un puisard a rencontré le niveau archéologique à la profondeur de 1,50 m (à cause de remblais accumulés à cet endroit). Il a été recueilli :

6 tessons de céramique grossière de 6 à 14 d'épaisseur comprenant : un tesson avec semis d'impressions unguéales et départ d'une anse brisée (fig. 11, $\mathrm{n}^{0} 1$ ), un tesson à cordon digital (fig. 11, n 4), 2 tessons à impressions sur la lèvre (fig. 11, nos 2,3 ) et 2 débris de rebords banaux.

3 tessons lournés récents;

2 éclats de silex bruts, dont un roulé, probablement paléolithique. 
Les Bas de Sainte-Radégonde. Site $う$

Dans l'axe du passage commun compris entre les nos 44 et 46 de la rue Paul-IIuet (cadastre AT 43), soit à $17 \mathrm{~m}$ du point $\mathrm{n}^{\circ} 3$, une tranchée d'adduction d'eau a recoupé, en octobre 1980 , à $0,80 \mathrm{~m}$ de la surface du sol, une fosse de 0,25 à $0,30 \mathrm{~m}$ de profondeur ; la surface ainsi mise au jour (environ un demi-mètre carré) a livré :

\section{Céramique "grossière».}

2 portions de grande jarre à panse galbée et col légèrement éversé, surface noirâtre présentant des traces d'ébauchoir horizontales, cordon à décor d'aspect torsadé et légères impressions digitales sur le bord interne ne constituant sans doute pas un décor intentionnel (fig. $10, \mathrm{n}^{\circ} 3$ ) ; D.0. : 460 ; D.C. : 435 ; D.P. : 465 ; e. : 7 à 12,16 au cordon ;

une portion de vase de même type, mais de profil moins sinueux, à surface marron clair avec passées noirâtres, décor d'une ligne d'impressions obliques obtenues à la spatulc (fig. $10, \mathrm{n}^{\circ} 1$ ) ; D.0. : 460 ; D.C. : 435 ; D.P. : 465 ; e. : 7 à 12,16 au cordon ;

une demi-douzaine de tessons de 6 à 12 d'épaisseur présentant des décors de cordons ou d'impressions digitales se rapportant à des types déjà décrits (fig. 10, no 2 ) ;

une vingtaine de tessons banaux.

2. Céramique fine, lustrée, noirâtre.

un débris de petit col éversé ; e. : 6 à 7 .

La Grange Liténard. Site 6

A $270 \mathrm{~m}$ à l'est des points précédents, au bord du chemin départemental no 21 de Chinon à Loches, le creusement des fondations de la maison Letellier (cadastre AW 73), en février 1954, amena la découverte d'une villa gallo-romaine étudiée par J. Zocchetti, R. Mauny et A. Héron (1959), qui signalent une occupation pré-romaine attestéc par des "silex et poterics grossières" sous les substructions. Ces trouvailles comprennent :

un tesson à pâte noirâtre, non lissée, provenant d'unc jarre de même type que les précédentes, décorée d'un cordon à impressions digitales (fig. $11, \mathrm{n}^{0} 5$ ) ; D.0. : 300 ; e : 8 à 10,13 au cordon ; un tesson à pâte noirâtre plus fine et légèrement lustrée, provenant d'une grande terrine (fig. $11, \mathrm{n}^{\circ} 6$ ) ; D.0. : 295 ; e : 9 à 10 ;

un éclat de silex brut et un grattoir aménagé sur une tablette de nucléus.

\section{La Grange Liénard (ou les Coutières). Site 7}

A $170 \mathrm{~m}$ au nord-nord-est du point précédent, à $80 \mathrm{~m}$ au nord du chemin départemental no 21 (terrain Toussaint, cadastre AW 99), M. J. Zocchetti a récolté, à l'occasion de la construction d'un garage, à une profondeur de $0,80 \mathrm{~m}$, une trentaine de tessons se répartissant comme suit :

1. Céramique "grossière 》. 22 tessons de 6 à 15 d'épaisseur. La meilleure pièce est une portion de vase sub-caréné à bord rentrant, décoré d'une ligne d'impressions digitales à 

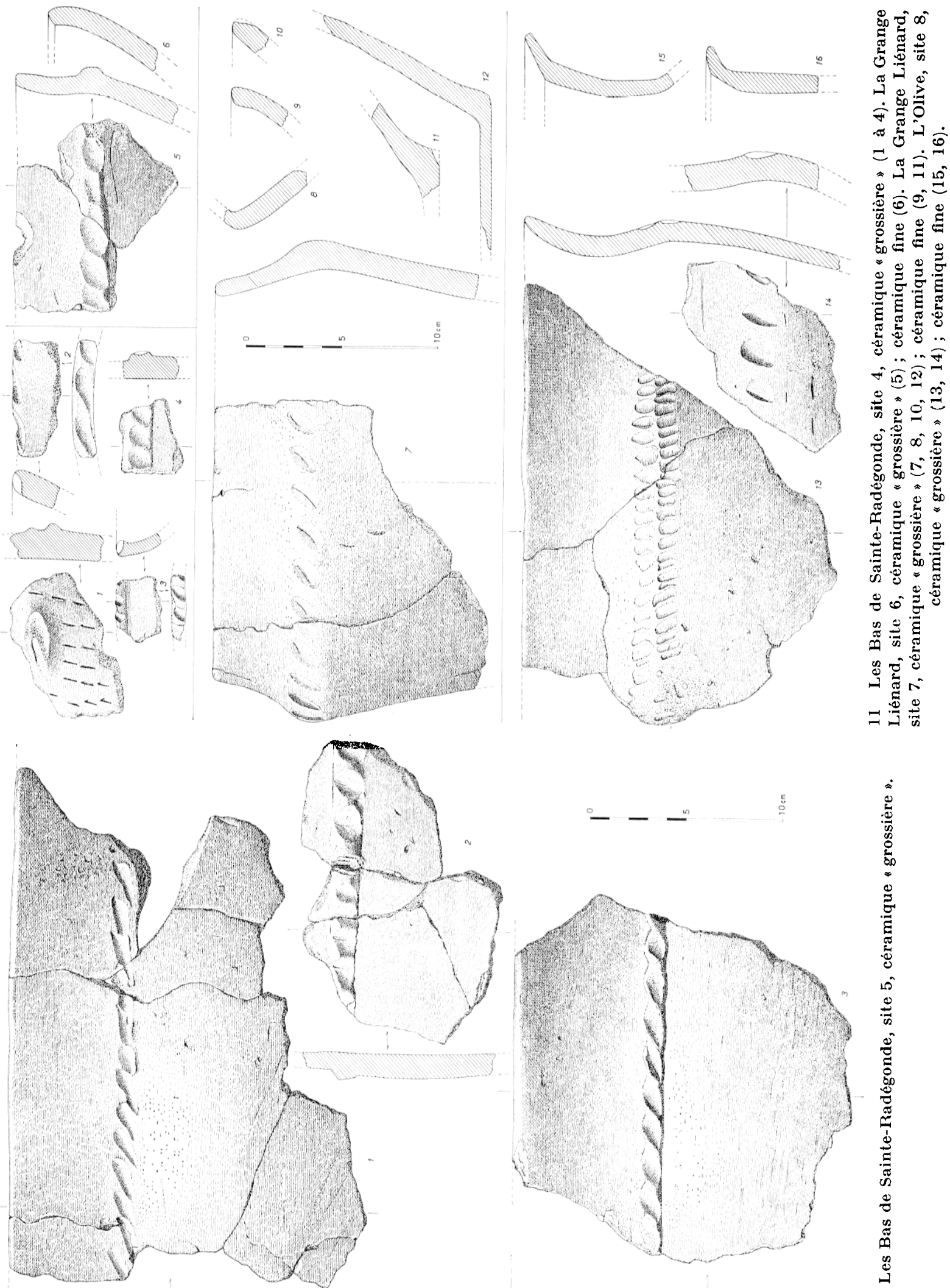

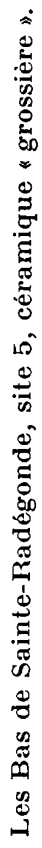

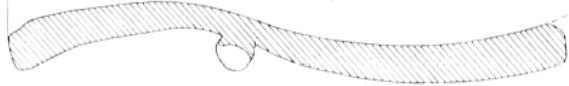


la carène (fig. 11, n 7) ; D.0. : 186 ; D.P. : 230. A noter également un fragment de fond plat avec une partic du flanc (fig. 11, no 12) et un débris d'épaule avec départ de col éversé pouvant provenir d'un vase bi-tronconique (fig. $11, \mathrm{n}^{0} 8$ ).

2. Céramique fine, lustrée, noirâtre : 8 tessons de 6 à 8 d'épaisseur, dont un bord de bol ou de terrine (fig. 11, no 9 ) et un débris de fond plat avec départ de flanc très oblique (fig. 11, $\mathrm{n}^{0} 11$ ).

\section{L'Olive. Site 8}

A $700 \mathrm{~m}$ à l'est des Bas de Sainte-Radégonde, des travaux effectués dans la cour de la maison Enjolras (cadastre BG 15), en mai 1968, ont livré, à environ $1 \mathrm{~m}$ de profondeur :

1. Céramique "grossière»: une portion de jarre à panse galbée et col éversé, à surface noirâtre, peu rugueuse, décorée à l'épaule de 2 rangées d'impressions irrégulières (fig. 11, no 13$)$; D.O. : 300 ; D.C. : 280 ; I).P. : 300 ; e. : 7 à 10 ; un débris d'un autre vase de même type, à décor digital sur l'épaule (fig. $11, n^{\circ} 14$ ); e. : 10 à 13 et 2 tessons banaux ; e. : 8 .

2. Céramique fine, lustrée, noire : un fragment de col évasé à bord déjeté et lèvre biseautée intérieurement (fig. $11, \mathrm{n}^{\circ} 15$ ) ; e. : 6 à 9 et un fragment de col cylindrique à bord et lèvre de même type (fig. 11, $\mathrm{n}^{0} 16$ ); D.0. : 400 ; e. : 7 à 10.

\section{$* *$}

C'est d'abord une impression d'homogénéité qui se dégage de l'ensemble des découvertes. Bien que les explorations réalisées en certains points ne revêtent qu'une valeur de sondages, il ne semble pas douteux que nous soyons en présence d'une série de points d'occupation relevant d'une même nappe de peuplement.

Les circonstances des découvertes n'ont pas permis toutes les observations de structure désirables, mais l'étendue de certaines aires archéologiques, notamment allx Bas de Sainte-Radégonde, suggère de véritables niveaux d'habitat et la présence de débris de pisé indique des constructions en torchis.

L'industrie lithique, si bien représentéc dans mainte fosse hallstattienne, est ici curieusement rare, encore ne peut-on écarter les risques d'introductions accidentelles.

La qualité générale de la céramique reflète un choix judicicux de la matière première et une technique consommée de façonnage et de cuisson. La céramique dite "grossière » - certains auteurs préfèrent "commune», "domestique» ou "utilitaire» - par opposition à la catégoric suivante reste une excellente production, solide et relativement soignéc.

Malgré l'état général fragmentaire, l'essentiel des formes et décors peut être présumé : céramique "grossière " : grandes jarres (dites aussi "vases à provisions") à fond plat, panse galbée et bord légèrement éversé, atteignant $60 \mathrm{~cm}$ de hauteur et $50 \mathrm{~cm}$ de diamètre, bols, terrines et jattes, le tout à décors en majorité digitaux ou, moins fréquemment, impressionnés à la spatule;

céramique fine lustrée : coupelles surbaissées à ouverture très évasée et fond ombiliqué, bols, terrines, jattes et quelques urnes à col évasé ou sub-cylindrique, grand dolium 

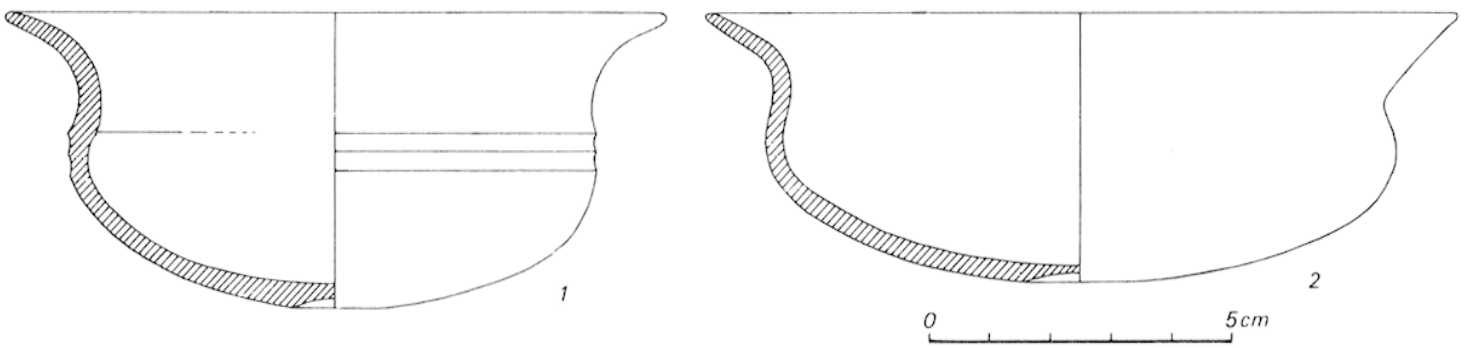

12 Profils comparés des coupelles de Chinon (1) et du tumulus de Chenevières à Savigny-en-Septaine (2) (d'après un dessin de P. Bailly).

exceptionnel de $80 \mathrm{~cm}$ de diamètre. Les décors dominants sont à cannelures parfois internes (sur les jattes); il est malheureusement difficile de déterminer la nature exacte du décor interne des coupelles, réduit à l'état de traces : peinture ou application de pâte ou de lamelles?

L'attribution d'ensemble au Premier Age du Fer ne soulève pas de difficultés. Il est moins aisé de préciser davantage, surtout en l'absence de tout élément métallique. L'ensemble de la céramique "grossière" est peu caractéristique. En s'en tenant aux formes, abstraction faite des caractères des pâtes, les grandes jarres à décors digitaux se retrouvent dans tout le Hallstatt et le Bronze final, voire plus haut : le dépôt de la fin du Bronze moyen de Chéry, par exemple, était contenu dans un vase de ce type. En ce qui concerne la céramique fine, les bols, jattes et terrines, souvent à cannelures internes, abondent dans les nécropoles des Champs d'Urnes du Bronze final, pour ne parler que de celles des Marais de Saint-Gond étudiées par B. Chertier. Il en va de même des urnes à col tronconique, évasé ou cylindrique et lèvres déjetées ou moulurées; qu'il suffise de citer les Champs d'Urnes orléanais (Férolles, Tigy) ; là aussi, les décors à cannelures sont largement répandus. Plus spécifiques semblent les coupelles, représentées par plusieurs exemplaires de profils presque superposables (fig. 7, nos 1 à 3). De telles formes n'abondent pas dans les ensembles décrits jusqu'ici. Il existe cependant une réplique assez fidèle en Berry, dans un des petits tumulus de Chenevières, à Savigny-en-Septaine, fouillés par P. de Goy à la fin du siècle dernier. La pièce mesure $124 \mathrm{~mm}$ de diamètre à l'ouverture et $41 \mathrm{~mm}$ de hauteur; le fond présente un ombilic d'environ $20 \mathrm{~mm}$ de diamètre et 1 à $2 \mathrm{~mm}$ de profondeur (fig. 12). P. de Goy se montrait frappé de son "galbe élégant" et de sa perfection au point de la croire faite au tour. Ce vase actuellement conservé au musée du Berry à Bourges (no 907.47.16) se trouvait dans une sépulture à inhumation, accompagné de quelques objets métalliques : un bracelet de bronze filiforme, une boucle ou anneau de bronze et un crochet en fer. N. Freidin fait entrer cette sépulture dans un groupe de tumulus berrichons datables du Hallstatt moyen (environ 650-550). Une telle attribution pour le matériel chinonais ne semblerait infirmée par aucun élément particulièrement précoce ni tardif.

Les connaissances sur les habitats hallstattiens dans les pays de la Loire moyenne ${ }^{2}$,

2 En marge de ces régions, l'important matériel céramique rencontré à Puiseaux (Loirel) est à rappeler ici on raison de ses relations générales avec celui de Chinon (Srmoxin 1983). 


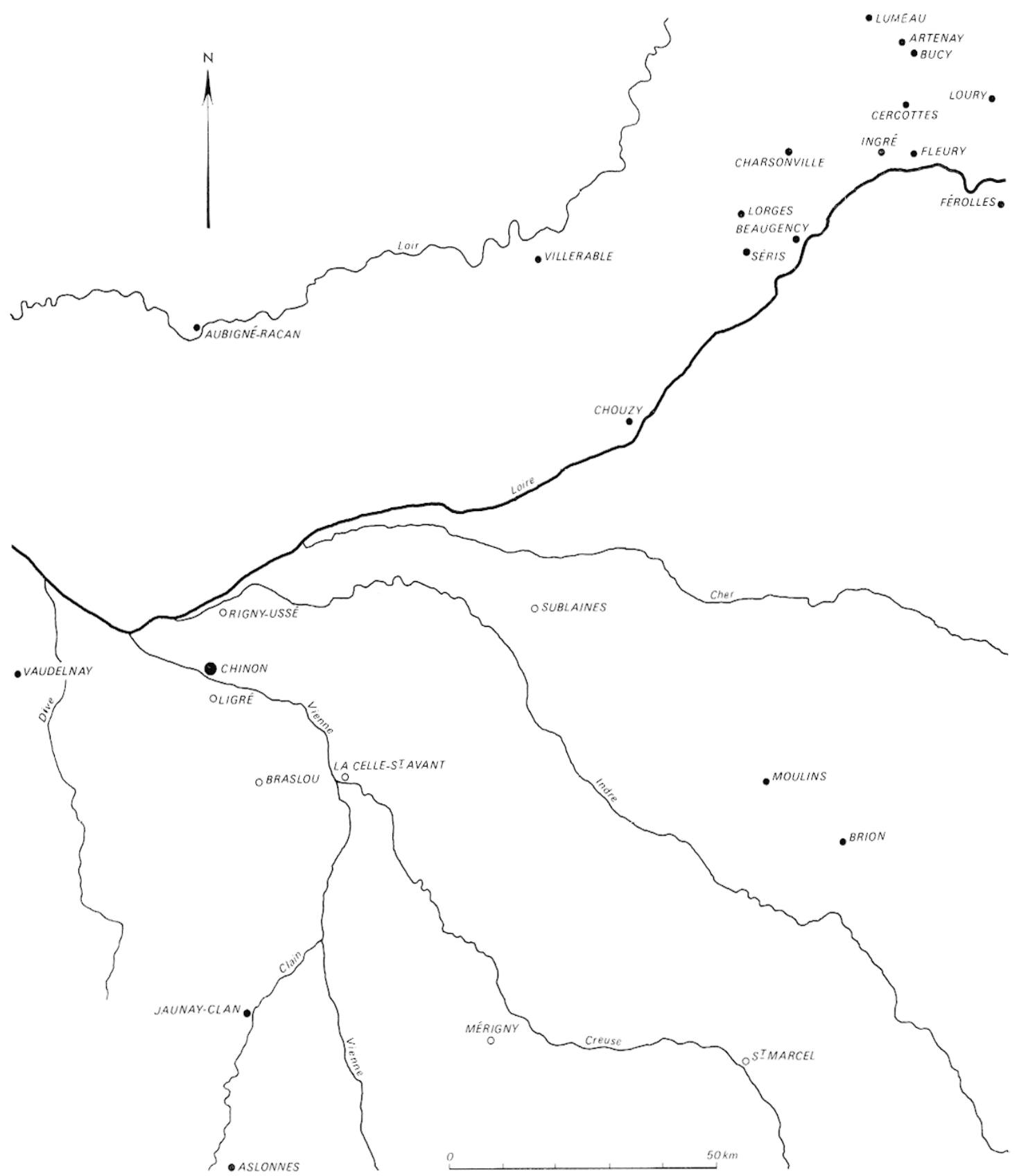

13 Sites d'habitats hallstatliens des régions de la Loire moyenne (points noirs) el quelques sites funéraires ou douteux (points blancs!.

qui se réduisaient à fort peu de choses voici une vingtaine d'années, se sont considérablement enrichies, comme en témoignent l'inventaire et la carte ci-après (fig. 13) :

1. Artenay (Loiret), à l'ouest de la sucrerie - Fosse avec céramique Hallstatt final/Tène ancienne et 1 fibule en bronze, à bouton. Bibl. : de Kısci 1978, p. 285.

2. Aslonnes (Vienne), Camp Allaric.

a) Occupation Bronze final/Hallstatt ancien (630 B.C.). Vestiges de cabanes adossées 
au rempart, avec matériel céramique très important : jarres, bols, jattes, terrines, urnes, etc. Décors d'impressions digitales, unguéales et diverses sur la céramique commune ; incisions, cannelures, impressions de cercles oculés, peinture rouge sur la céramique fine; fusaïoles. Objets de bronze (perle, épingle), industrie osseuse (poinçons), bracelets en terre cuite et autres objets de parure. Faune abondante (bovidés, suidés, cervidés).

b) Occupation Hallstatt final. Céramique très abondante : jarres, bols hémisphériques et à fond plat, urnes globuleuses à col éversé, vases à pied, etc. Nombreux décors géométriques au graphite sur la céramique fine. Objets de bronze (épingle, fils torsadés...) et de fer (pointe de lance, fragment de fibule...). Bracelets en lignite.

Bibl. : Pautreau 1976.

3. Aubigné-Racan (Sarthe), Camp de la Butte de Vaux. Habitat fouillé sur une douzaine de mètres carrés. Céramique grossière (en majorité) : grandes jarres, bols, jattes ; décors de cordons digités et torsadés. Tessons de céramique fine, lustrée, à décors de cannelures horizontales et d'incisions en dents de loup. Industrie lithique assez abondante (grattoirs, hache polie, nucléus, lames, éclats...). Bronze : talon de javeline. Des analyses G 14 ont donné des datations de 630,480 et 360 B.C.

Bibl. : Rioufreyt et Lambert 1977 ; Lambert et Rioufreyt 1981, p. 44 ; Freidin 1982, p. 523 ; Lambert et Rioufreyt 1983, p. 40-41.

4. Beaugency (Loiret), Les Hauls de Lulz. Céramique grossière : tessons et petit vase à décors d'impressions unguéales et d'incisions linéaires et en chevrons. Céramique fine lustrée noire : jattes ou terrines (?) et débris de bords de types divers. 1 fragment de fer. Faune : bœuf, porc.

Bibl. : Freidin 1982, p. 390-391.

5. Beaugency (Loiret), Vernon. 2 foyers ou fonds de cabanes et 5 fosses ayant livré un matériel céramique homogène avec une quinzaine de formes restituables ou présumables. Céramique grossière : grande jarre, vases cylindroïdes, bols et terrines à bord légèrement rentrant, jatte à bord sub-vertical, coupelle tronconique; décors digitaux et unguéaux sur les bords et les lèvres, cordons torsadés. Céramique fine : bol et jatte à bord sub-vertical, gobelet. Faune : mandibule de cheval, dents de porc, chèvre ou mouton.

Bibl. : Freidin 1982, p. 391.

6. Bordes (Les) (Loiret), le Garage. Habitat et fosses. Céramique abondante mais très fragmentée, tessons à engobe rouge, décors d'impressions digitales et de cannelures. Bibl. : Picard 1972, p. 316 ; Freidin 1982, p. 392.

7. Bordes (Les) (Loiret), le Ravoir. Vestiges d'un four de potier avec nombreux fragments de céramique généralement grossière, notamment un bol presque tronconique, une coupelle en forme de calotte, des fragments de fonds plats et de bords éversés, quelques tessons à décors d'impressions digitales et de cordons torsadés. Hallstatt ancien/moyen.

Bibl. : Nouel 1952 ; ID. 1957 ; Freidin 1982, p. 392.

8. Brion (Indre), les Grandes Chapelles. Site occupé du Néolithique à La Tène. Le Hallstatl est représenté par une épée complète du type de Güdlingen, 3 fragments d'autres épées, une bouterolle du type de Prüllsbirkig de P. Schauer, 1 bracelet à bossettes, 1 pointe 
de flèche en fer du type le Bourget et des tessons peu caractéristiques (petite jatte?, tesson à décor torsadé?, fonds de jarres ou d'urnes?).

Bibl. : Gharbonnier 1953 ; Buchsenschutz, Willaume et Gablin 1979 ; Freidin 1982, p. 372-373.

9. BucY-Le-Ror (Loiret). Fosse. Quelques tessons de céramique fine, 1 fusaïole en terre cuite, 1 fragment d'armille en bronze.

Bibl. : Groupement archéologique Artenay 1973 ; Picard 1974, p. 301 ; Freidin 1982 , p. 393.

10. Cercottes (Loiret), en forêt d'Orléans. Fosse contenant plus de 200 tessons, dont une vingtaine utilisables. Céramique grossière : tessons à décors d'impressions digitales et d'incisions linéaires. Céramique fine : 1 urne à panse galbée, col tronconique et décor de cannelures, fragments de bols ou jattes à décor de cannelures, portion inférieure de vase polypode. Industrie lithique : 1 molette.

Bibl. : Picard 1974, p. 301-303 ; Willaume 1975 ; Freidin 1982, p. 393-394.

11. Charsonville (Loiret), Montapeine. Fosse et fond de cabane. Céramique grossière : une coupelle à lèvre crénelée, des fragments de jarre ct de bol, un fond plat (de jatte?).

Bibl. : Quatrehomme 1960 ; Freidin 1982, p. 394.

12. Chouzy-sur-Crsse (Loir-et-Cher), le Pressoir Berry. Fond de cabanc (?) signalé par L. Guignard dans une publication malheureuscment peu claire ${ }^{3}$. Une centaine de tessons appartenant à une dizaine de vases. Círamique grossière : fragments de jarres à incisions obliques et à cordons à décors digitaux et torsadés. Céramique fine, lustrée : au moins un débris de col cylindrique. A remarquer une grande jatte de $480 \mathrm{~mm}$ de diamètre (figuréc, mais non décrite). Industrie lithique : un grattoir, une "pointe», "quelques silex à peine ébauchés ", une molette.

Bibl. : Guignard 1909 ; Freidin 1982, p. 384.

13. Férolles (Loiret), le Parlerre Est. Vestiges d'habitat démantelé en surface. Nombreux tessons. Céramique grossière : débris de grandes jarres à cordons, décors digitaux et unguéaux. Céramique fine : fragments de terrines à décors de cannelures, fragment d'urne bulbeuse à décor de grecques, 1 tesson présentant les traces d'un décor géométrique pcint ou appliqué.

Bibl. : Cordier 1977 ; Freidin 1982, p. 394-395.

14. Fleury-t.es-Aubrais (Loiret), hôpital psychialrique. Fosse. Céramique grossière abondante : fragments de grandes jarres, jattes ou terrines à décors d'impressions digitales et unguéales sur les bords et sur les lèvres leur donnant un aspect festonné. Cćramique fine rare : fragments d'urnes galbécs à col cylindrique ct de bol ou jatte ; décors de cannelures ; 1 fusaïole en terre cuite.

Bibl. : Nouel 1969 ; Freidin 1982, p. 395.

3 Lors d'une enquête sur place en 196.1, nous avons pu préciser l'emplacement de la découverle gràce à un vieil habitant de Chouzy (M. Datet) ayant connu les ouvriers cités par Guignard : une carriere abandonnée siluéc au bord du chemin vicinal $\mathrm{n}^{\circ} 5$, à $100 \mathrm{~m}$ au nord de la dernière maison du Pressoir-Berry (BE 125 du cadastre de 1964). 
15. Ingré (Loiret). Vestiges d'habitat. Tessons, parmi lesquels : 1 fragment de jatte en céramique grossière, à cordon torsadé, 1 tesson à incisions obliques, 2 tessons à cannelures, 1 débris de vase caréné à décor incisé (incisions linéaires parallèles et perpendiculaires). Débris d'argile cuite.

Bibl. : Freidin 1982, p. 396.

16. Jaunay-lían (Vienne), Sous-Clan, dans la vallée du Clain. Fond de cabane. Nombreux tessons appartenant à 36 vases au moins : jarres, dont une grande à décor unguéal (1).0. : $260 ;$ H. : 375)), terrines, jattes à décors incisés (rectangles et grecques notamment), faisselle, gobelet à anse de profil comparable à celui de (hinon (fig. 5, no 4). Industrie lithique : 9 éclats. Faune : dents de chèvres. Hallstatt moyen (env. 600 B.C.?)

Bibl. : TAILlet 1956.

17. Longes (Cher). Fond de cabane avec céramique non tournée; "certains tessons présentent formes et décors caractéristiques du Hallstatt».

Bibl. : de Kısch 1978, p. 281.

18. Louny (Loiret), les Carrières de Loury. 2 fosses avec de nombreux tessons de céramique grossière et de céramique fine ; parmi ces derniers, 1 coupelle à lèvre crénelée. Quelques silex taillés. Débris de charbon de bois et cendres.

Bibl. : Baratin 1966 ; Nouel 1967.

19. Lumeau (Eure-et-Loir), Domainville. Vestiges d'habitats dispersés en surface; plus de 200 tessons. Céramique grossière en majorité : débris de bols ou de terrines et de fonds plats; décors d'impressions digitales et unguéales, fréquemment sur les lèvres, leur donnant un aspect festonné. Quelques tessons de céramique fine, notamment un fragment de gobelct à décor peint ou appliqué (traces rectilignes et en zigzag). 2 fibules en fer à arbalète (hallstattiennes?). Vestiges gaulois, gallo-romains et médiévaux sur le même site ${ }^{4}$.

Bibl. : Picard 1974, p. 300.

20. Moulins-sur-Céphons (Indre). Enceinle des Châteliers. Rempart de terre à ossature de poutres formant caissons, daté du Bronze final/Hallstatt.

Bibl. : Duval et Buschenschutz 1979.

21. SÉris (Loir-et-Cher), les Grands Réages de Dugny.

- 1er fond de cabane. Tessons provenant d'une dizaine de vases, parmi lesquels des jarres à cordon à décor torsadé (les autres formes sont difficilement restituables), décors à cannelures et, incisions rectilignes et curvilignes, 1 tesson peint. Industrie lithique : 1 lame de silex. Faune : dents de bœuf et de porc ou de sanglier. Hallstatt moyen.

Bibl. : Quatrehomme et Lhermitte 1968. Freidin 1982, p. 385.

- $2^{\mathrm{e}}$ fond de cabane (à $40 \mathrm{~m}$ du précédent). Une trentaine de tessons provenant d'au moins 23 vases. Céramique "grossière" : grandes jarres (H. : 285 et 370 ) à décors digitaux sur cordons et sur les lèvres et 2 bols. Céramique fine $: 1$ bol à panse sub-carénée, des tessons à décors de lignes incisées et à peinture. Bronze : fragments d'un bracelet à incisions

4 Nos remerciements a 11 . lzobert Plessis, decouvreur de ce site, qui nous fit parvenir ses trouvailles el observations en 1969 par le canal de l'abbé Nouel. 
transversales et bossettes termmales, anneau, petite lame. Silex : grattoir, couteau, percuteur et quelques éclats. Faune : débris osseux de chevreuil, cerf, sanglier, chèvre, mouton, lièvre. Hallstatt moyen.

Bibl. : Picard 1972 (alias Josnes); Dessphíe el Lhermite 1972; Freidin 1982, p. $385-386$.

22. Vaudelnay (Mainc-et-Loire), Fierbois. 6 fosses dont la seule explorable a livré la partic supérieure d'une petite urne noire, lustrée, à panse galbée, col éversé et fond rétréci, ornée d'incisions horizontales, quelques tessons dont 1 à engobe rouge. Restes de faune : cerf, bœuf, blaireau, oiseaux, mollusques. Au voisinage, 1 talon de javeline en fer.

Bibl. : Gruet 1952 ; Cornier et Gruft 1975, p. 277-278.

23. Villerable (Loir-et-Cher), les Grands Marais. Fosse, Environ 300 tessons. Céramique grossière : débris de jarres et de terrines; décors d'impressions digitales et de cordons d'aspect torsadé, lèvres festonnées. Céramique fine : petit gobelet, fragments de bols et de jattes à marli, décors d'incisions linéaires et de cannelures. Industrie lithique : 166 silex (grattoirs, lames retouchées, ćclats de haches polies, nucleus, etc.). Faune : 1 dent de bœuf. Hallstatt ancien (fouille Bailloud-Cordier). Nombreux vestiges céramiques de mêmes types provenant d'habitals démantelés dans les environs (les Grands Marais, les Terres Blanches, les Petils Marais, la Croix, Martigny, la Vallée Terreau...) (recherches (Ch. Motheron).

Il faudrait ajouter des trouvailles sporadiques de tessons ou de vases constituant des indices de passage ou d'occupation dans diverses localités : Assay, Beaugency, les Bordes, Lavardin 5 , Lorris, Martizay, Maves, Meung-sur-Loire, Montrichard, Nancray-surRimarde, Neuville-aux-Bois, Saint-Genou, Saint-Marcel (?) ${ }^{6}$, Selles-sur-Cher, Sublaines, Thenay, Tours... Les dragages de la Loire ont également livré de nombreux tessons et parfois des vases complets, notamment à Amboise, Beaugency, Baule, Meung-sur-Loire. Malheureusement, il est souvent difficile, devant des vestiges céramiques dépourvus de contexte, de trancher entre le Bronze final et le Hallstatt.

Ce tour d'horizon régional n'apporte pas de relations adéquates ni précisément synchroniques aux habitats de Chinon. Ceux-ci, malgré l'importance de l'implantation qu'ils supposent, font figure à ce jour d'isolat entre les sites du Poitou (Jaunay-Clan, $\Lambda$ slonnes), de la vallée du Loir (Aubigné-Racan), de celle de la Loire (Chouzy) et de la Beauce, où l'occupation hallstattienne paraît largement répartic. Des sites comme ceux de Séris et de Chouzy, proches de la Loire, semblent très comparables aux découvertes de Chinon mais, dans le premier cas, les inventeurs n'ont pu affiner la datation et, dans le second, la documentation reste par trop insuffisante.

L'avenir nous livrera-t-il les sites funéraires correspondant à ces habitats? Pour

5 F. Auhin (1915) figure des tessons à impressions pouvant appartenir à l'Age du Bronze ou au Hallstatt recueillis sur "une petile plale-forme prolongeant le talus " de la Bulle de Labardin, en mélange, semble-l-il, avec de l'industrie néolithique el des tessons gallo-romains.

6 On reste perplexe devant les vases a cordons digilés ou crenelés figures par E. Uubert comme provenant d'une couche supérieure de la grotte de la Garenne en compagnie d'aulres objets pour le moins héléroclites. 
l'heure, nous ne connaissons dans le voisinage que les tumulus de Rigny-Ussé à une dizaine de kilomètres au nord et ceux de Braslou à une vingtaine de kilomètres au sud-est. Dans les deux cas, les fouilles remontent au début du siècle, les comptes rendus sont assez sommaires et le matériel à peu près inexistant. Un autre tumulus en a été rapproché, les Roches-Saint-Paul à Ligré, mais les renseignements à son sujet sont si peu convaincants qu'on peut aussi bien penser à une tombelle néolithique. Enfin, la sépulture à incinération de la Celle-Saint-Avant, près du confluent Creuse-Vienne se place à une phase légèrement antérieure (Hallstatt $B$ de Reinecke = Bronze final III b de Hatt).

L'activité métallurgique des Hallstattiens de Chinon reste un problème entier. Hormis quelques débris de mâchefer - peut-être intrusifs - aucun élément métallique n'a été recueilli. Un gîte à minerai de fer d'extraction facile existe à 3 ou $4 \mathrm{~km}$ au nord-est, dans les Bois de Grammont, mais rien ne prouve son exploitation à une époque aussi haute et, en tout cas, on ne saurait y voir un élément déterminant de l'implantation humaine qui nous occupe. De même, rien ne permet encore d'attribuer à une époque antérieure au Gallo-Romain les nombreux vestiges sidérurgiques que recèle la forêt de Chinon.

Bien que dépourvus de prédécesseurs sur les licux mêmes - pourtant si propices à l'habitat - les Hallstattiens de Chinon font suite à une occupation ou à une circulation locales bien attestées par de nombreuses découvertes de l'Age du Bronze : pointe de lance et rapière draguées dans la Vienne à Chinon même, dépôt d'Azay-le-Rideau, trouvailles d'objets divers à Azay-le-Rideau, Cheillé, Marçay, Panzoult, Trogues, Parçay-sur-Vienne... Sur l'époque suivante, par contre, nous ne possédons que fort peu de témoignages. Le Dictionnaire Archéologique de la Gaule mentionne deux statères d'or, quatre deniers d'argent et deux petits bronzes à la légende Contovros provenant de Chinon, sans plus de précision. Que de telles trouvailles eussent été les bienvenues à l'emplacement du château, lequel aurait succédé à un oppidum gaulois, selon une tradition qui semble reposer davantage sur la séduction du site que sur de réelles preuves archéologiques : "L'oppidum de Caino était ce lieu de refuge pour les populations celtiques de la rive droite de la Vienne...». Admirable prémonition de l'historien chinonais G. de Cougny à une époque où l'on ignorait tout du village "celtique" qui, à quinze cents mètres du majestueux promontoire, était promis au rôle de berceau de la cité chinonaise.

Gérard Cordien

\section{Bibliographie.}

Amis du Vieux Ghinon. Chronique archéologique et historique de l'arrondissement de Chinon. Bull., 1967, p. $140 ; 1968$, p. 251 ; 1981, p. 721.

Aubin (E.). La Butle ou "tombelle» du château de Lavardin dans Bull. Soc. archéol. scient. lill. Vendômois, 1915, p. 46-53.

Baratin (J.-Fr.). Découverle dans une sablière à Loury (Loirel) dans Bull. Irim. Soc. archéol. neuvilloise, 1966, 12, p. 1-4. 
Buchsenschutz (C.), Willaume (M.) et Gablin (P.). Le site Bronze final-Premier Age du Fer des Grandes Chapelles à Brion (Indre) dans Bull. Soc. préhist. fr., 1979, p. 408-420.

Charbonnier (0.). Découverle d'une épée de bronze hallstattienne près de Brion (Indre) dans Congrés préhist. Fr., Strasbourg, 1953, p. 198-203.

Chertier (B.). Les nécropoles de la civilisalion des Champs d'Urnes dans la région des Marais de Saint-Gond (Marne). Paris, 1976.

Condier (G.) et Gruet (M.). L'Age du Bronze et le Premier Age du Fer en Anjou dans Gallia Préhisl., 1975, p. 157-287.

Cordier (G.). Les tumulus hallslaltiens de Sublaines (Indre-el-Loire), I, Eitude archéologique dans L'Anthr., 1975, p. 451-482, 579-628.

Cordier (G.). Les Champs d'Urnes en Orléanais dans Rev. archéol. Loiret, 1977, p. 9-39.

Cordier (G.). La grotte funéraire hallstattienne de la Roche Noire à Mérigny (Indre) dans L'Anthr., 1978, p. 199-220.

Cordonnier-Détrie (P.). Informations. Gallia, 1955, p. 161-166.

Cougny (G. de). Chinon et ses environs. Tours, 1898.

Despriḱe (J.) et Lhermite (J.). Découverle d'une fosse hallstattienne sur la commune de Séris (Loir-et-Cher) dans Rev. archéol. Centre, 1972, p. 112-125.

Duval (A.) et Buchsenschutz (0.). Sondages sur un rempart prolohistorique à Moulinssur-Céphons (Indre) dans Groupe IIisl. Archéol. Buzançais, 11, 1979, p. 19-28.

Freidin (N.) The Early Iron Age in the Paris Basin, IIallslalt $C$ and D. Oxford, 1982.

Goy (P. de). Sépultures antiques en Berry dans Mém. Soc. Antiq. Centre, 1887-1888, p. 47-61.

Groupement archéologigue n'Artenay. Fouille de sauvelage de Bucy-le-Roi dans Bull., 1973, 1, p. 8-9.

Gruet (M.). Une faunule du Qualernaire récent à Fierbois (près Doué-la-Fonlaine) dans Bull. Soc. El. scient. Angers, 1952, p. 31-35.

Guignard (L.). Trouvaille au Pressoir-Berry, à Chouzy-sur-Cisse (Loir-el-Cher) dans Rev. préhisl. ill. Est Fr., 1909, p. 41-46.

In bert (E.). Le Bas Berry, IIsloire et Archéologie du déparlement de l'Indre, II, le canton d'Argenton. Châteauroux, 190\%.

KIsch (Y. de). Informations Gallia, 1976, p. 311-329.

Kısсн (Y. de). Informalions Gallia, 1978, p. 261-293.

Lambert (Cl.) el Rioufreyt (J.). L'habitat rural antique dans le Maine el la pholographie aérienne dans La Mayenne, Archéol. Hist., 1981, p. 33-98.

Lambert (Cil.) et Rroufrey'T (J.). Les temps préhisloriques dans La Sarthe des origines à nos jour's, Saint-Jean-d'Angély, 1983, p. 21-j4.

Nouri (A.). Les T'umulus el les Anliquilés de la Forêl d'Orléans (Inspection de Lorris) (Loirel) dans Bull. Soc. préhist. fr., 1952, p. 330-341. 
Nouel (A.). Les découverles des Ages du Bronze el du Fer dans le département du Loirel dans Bull. Soc. préhisl. fr., 1957, p. 307-319.

Novel (A.). Les découvertes des Ages du Bronze et du Fer dans le déparlement du Loirel (3e Supplémenl) dans Bull. Soc. préhist. fr., 1967, p. ccxiv-ccXvirI.

Nouel (A.). Un fond de cabane de l'Age du Fer à Fleury-les-Aubrais (Loirel) dans Bull. Soc. archéol. hisl. Orléanais, 1969, p. 409-413.

Pautreau (J.-P.). Le Camp Allaric, commune d'Aslonnes (Vienne), premiers résullats dans L'Anthr., 1976, p. 389-429.

Picard (G.-Ch.). Informalions Gallia, 1972, p. 313-332.

Picard (G.-Ch.). Informations Gallia, 1974, p. 299-317.

Quatrehomme (Fr.). Poteries protohistoriques trouvées à Charsonville el Meung-sur-Loire (Loirel) dans Bull. Soc. préhisl. fr., 1960, p. 309-312.

Quatrenomme (Fr.). Poleries el fragments trouvés en Loire en face de Baule dans Bull. Soc. préhist. fr., 1965, p. XXXVI-XLII.

Quatrehomme (Fr.) et Lhermite (J.). Un fond de cabane du débul de l'Age du Fer, commune de Séris (Loir-el-Cher) dans Bull. Soc. préhist. fr., 1968, p. 206-212.

Rioufreyt (J.) et Lambert (Cl.). Le camp de la Bulle de Vaux à Aubigné-Racan dans Province Maine, 1977, p. 1-20.

Sacchi (D.) et Zocchetri (J.). Présence de céramique hallstaltienne dans le Chinonais dans Bull. Soc. Amis vieux Chinon, 1965, p. 469-472.

Sacchi (D.) et Zocchetrt (J.). Nouvelle découverte de céramique prolohislorique à Chinon dans Bull. Soc. Amis vieux Chinon, 1967, p. '2-4.

Séronie-Vivien (M.-R.). Introduction à l'étude des poteries préhistoriques. Le Bouscat, 1975.

Srmonin (D.). Céramique du premier Age du Fer à Puiseaux (Loirel) dans Bull. Soc. préhist. fr., 1983, p. 119-128.

Taillet (M.). La slation prolohistorique de Sous-Clan près Poitiers (Vienne) (Hallstaltien) dans Congr. préhisl. Fr., Poitiers, 1956, p. 895-913.

Willaume (M.). Sauvelage d'une fosse du premier Age du Fer à Cercottes, Loirel dans Rev. archéol. Nord Loirel, 1975, p. 17-20.

Zocchetti (J.), Mauny (R.) et Héron (A.). Découverle d'une villa gallo-romaine à Chinon dans Amis du vieux Chinon, 1959, p. 164-170. 